\title{
Three-dimensional analysis of the accuracy of optic and electromagnetic navigation systems using surface registration in live endoscopic sinus surgery*
} \author{
Po-Wen Cheng ${ }^{2,3}$ \\ ' Institute of Biomedical Engineering, National Taiwan University, Taipei, Taiwan \\ 2 Department of Otolaryngology, Far Eastern Memorial Hospital, Taipei, Taiwan \\ ${ }^{3}$ Oriental Institute of Technology, Taipei, Taiwan
}

Chih-Ming Chang ${ }^{1,2}$, Fu-Shan Jaw ${ }^{1,2}$, Wu-Chia Lo², Kai-Min Fang²,

Rhinology 54: 88-94, 2016

DOI:10.4193/Rhino15.131

*Received for publication:

May 16, 2015

Accepted: October 12, 2015

\begin{abstract}
Background: This study presents the first report in the same patients on the time efficiency of surface registration as well as the navigational accuracy using optic and electromagnetic tracking systems.

Methods: Thirty patients with bilateral chronic paranasal pansinusitis underwent endoscopic sinus surgery. After surface registration, the surgeries were performed on one side using optic navigation guidance and on the other side using electromagnetic navigation guidance. The intraoperative measurements performed included the time taken for the surface registration and surgical procedure on each side, as well as the navigation errors at the different locations.
\end{abstract}

Results: The time for surface registration was significantly longer in the optic navigation group than the electromagnetic group. A comparison of the navigation errors along the 3 axes showed that the deviation in the medial-lateral direction was significantly less than that in the anterior-posterior and cranial-caudal directions in the optic navigation group as well as the electromagnetic group.

Conclusions: The procedure for surface registration in both optic and electromagnetic guidance is efficient and convenient. The accuracy of both navigation systems is comparable and within acceptable ranges for clinical use. In addition, the best accuracy was measured in the medial-lateral direction compared with the other two axes.

Key words: surface registration, optic navigation, electromagnetic navigation, computer-aided surgery, navigation error

\section{Introduction}

Functional endoscopic sinus surgery has gained wide application in the management of sinus disease since the 1980s and has generally replaced traditional Caldwell-Luc surgery ${ }^{(1)}$. However, it is still associated with a definite risk for both intraoperative and postoperative complications ${ }^{(2)}$. The use of computer-aided surgery (CAS) technology was developed to assist surgeons in identifying anatomic landmarks during sinus surgery ${ }^{\left({ }^{(3)}\right.}$. It has been used since the 1990s, and intra-operative complications such as orbital and intracranial injuries can be prevented with the aid of real-time image guidance in association with endoscopy ${ }^{(2)}$.

The various commercially available CAS systems, including op- tical systems ${ }^{(4)}$, electromagnetic systems ${ }^{(5)}$, electromechanical systems ${ }^{(6)}$ and sonic tracking systems ${ }^{(7)}$, can be used to locate the exact position of a tracked instrument. Utilizing different principles, the systems are able to calculate the motion of the sensor of the instrument in relation to the reference frame on the patient and continuously display the relative three-dimensional position in real time on the monitor. In this manner, the images are used to guide the surgical planning and approach. In CAS, a correlation between a stored image data set (e.g. computed tomography (CT) or magnetic resonance imaging (MRI)) and specific landmarks in the surgical area is required. After the registration process, the navigation system provides updated positional information for each navigated instrument. Based on the anatomical landmarks ${ }^{(8)}$, fiducial markers ${ }^{(9,10)}$ or a surface 
matching method ${ }^{(11-13)}$, various registration algorithms have been developed. Anatomical landmark registration has been commonly used in a variety of practical situations, as it utilizes natural markers and is non-invasive ${ }^{(8)}$. The recently-developed surface registration procedure that aligns the unique facial contours is also appropriate for clinical use, because it can eliminate the problem of a long preparation time of the landmark matching with only slightly less accuracy ${ }^{(11-13)}$.

Comparisons of the optic and electromagnetic systems using fiducial marker-matching registration revealed a similar level of accuracy within $2 \mathrm{~mm}$ during sinus surgery ${ }^{(14)}$. However, comparisons between systems using surface registration have not been reported, either in live surgery or cadaveric dissection. Hence, the purpose of this pilot study is to investigate the anatomical precision of the two different navigation systems using surface registration in the course of live endoscopic sinus surgery on the same patients, and to share our experience with their use in clinical practice.

\section{Methods}

\section{Patients}

Thirty patients ( 24 men and 6 women) with chronic paranasal pansinusitis were enrolled in this study between January 2014 and December 2014. The severity of the disease was classified according to the Lund-Mackay CT scan classification systems of chronic rhinosinusitis. All patients were scored as Grade III-IV nasal polyposis according to the endoscopic grade system proposed by Meltzer et al. ${ }^{(15)}$. The total score for each case was greater than 15 in the Lund-Mackay CT scan classification system. We excluded patients younger than 20 years, patients with concomitant external sinus surgery, unusual soft tissue sensitivity or damaged tissue at the intended mounting area. We also excluded those with any circulatory disease of the skin that might make it prone to damage by pressure from the silicone contact pad of the head frame that is used as the navigation reference. Prior to surgery, physicians performed CT scans of the sinus area to obtain images at a $1 \mathrm{~mm}$ slice thickness. The image data were then transferred to the navigation unit using a compact disc. The same medical team performed bilateral endoscopic sinus surgery using the Medtronic S7 navigation system on all patients. The S7 system has both optic and electromagnetic tracking devices in one machine to facilitate the operation. We used the optic navigation system when performing one side endoscopic sinus surgery and the electromagnetic navigation system to assist the other side endoscopic sinus surgery in a random allocation through the use of a random number table. The test statistics measured intra-operatively in this study indicate the times taken for surface registration and the surgical procedure, as well as the navigation errors (NEs) along the 3 axes.

\section{Equipment set-up}

After the induction of general anesthesia, a head reference frame was attached to the patient's forehead using an elastic strap. The head frame was equipped with a headset patient tracker to provide a continuous point of reference for the navigation system. The head frame was placed at the center of the forehead and manipulated gently to ensure that it was attached securely, and that its position relative to the head would be maintained during the registration process and subsequent procedure. The localizer (i.e. the optic system camera or the electromagnetic field emitter) was set up before the surgery on each side. For optical tracking, the system camera triangulates the spatial coordinates of the optical markers on the tracked devices. The position the camera head is approximately $1.75 \mathrm{~m}$ from the patient reference, and the camera must have a clear line of sight to the optical markers. For electromagnetic tracking, a low-energy electromagnetic field emitter was fixed on a holder and attached to the operating table bed frame. The emitter holder was positioned at least $20 \mathrm{~cm}$ above the operating table and pointed directly at the patient's nose from a distance of $20 \mathrm{~cm}$. No part of the holder was extended into the emitter's electromagnetic field. All of the instrument tracker cables for intraoperative electromagnetic navigation were connected to the navigation unit ports.

\section{Surface registration}

A surface registration technique was used in both the optic and electromagnetic systems. Synergy Cranial Software was employed for the registration and measurement in both systems. The points that were used for the surface matching computer algorithm were distributed along various locations at the nose, forehead and orbital rim. After collecting a sufficient number of points on the scanned area for computer calculation, the threedimensional location information was automatically matched with the $\mathrm{CT}$ image. After completing the registration procedure, the correlation between the position of the instrument in the surgical field and the corresponding location on the CT images was established to allow real-time orientation during surgery. No additional registration processes were needed during the operation to adjust for anatomical drift. The time required for surface registration in both systems was recorded.

\section{Navigation error}

During the operation, a best effort was made to avoid moving or tilting the patient's head or the operating table. The bulb press testing was sometimes performed to check the integrity of the lamina papyracea. However, the head reference frame must remain attached and stable until navigation is complete to ensure an accurate navigational reading.

We cleared the abnormal sinonasal tissue on both sides to achieve adequate exposure of the skull base, lamina papyracea 


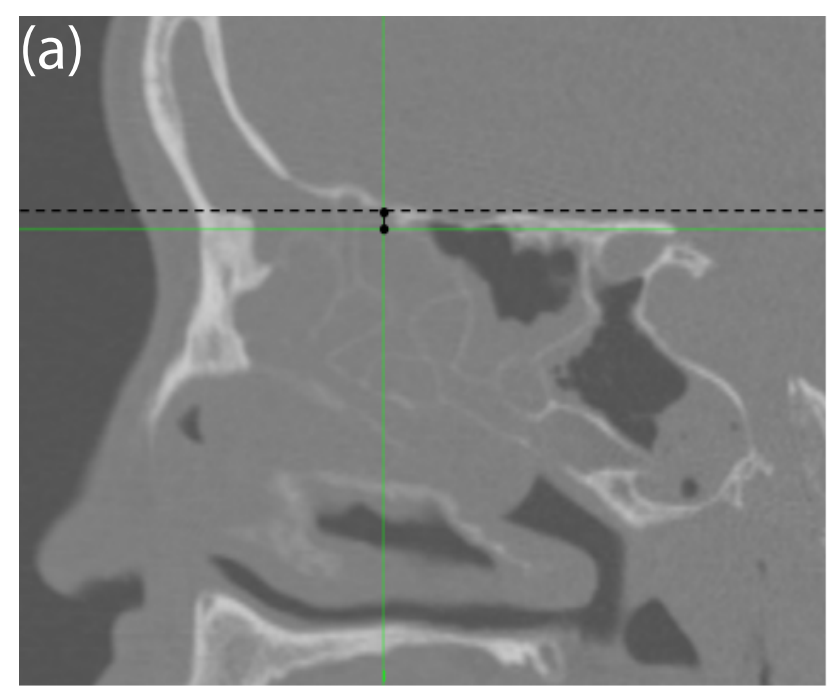

$\mathrm{AE}$

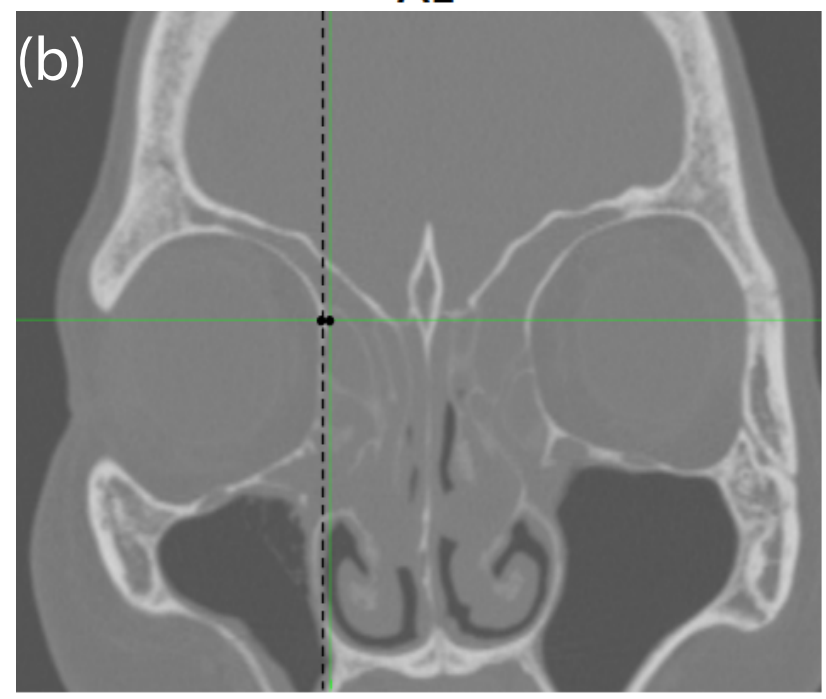

LP

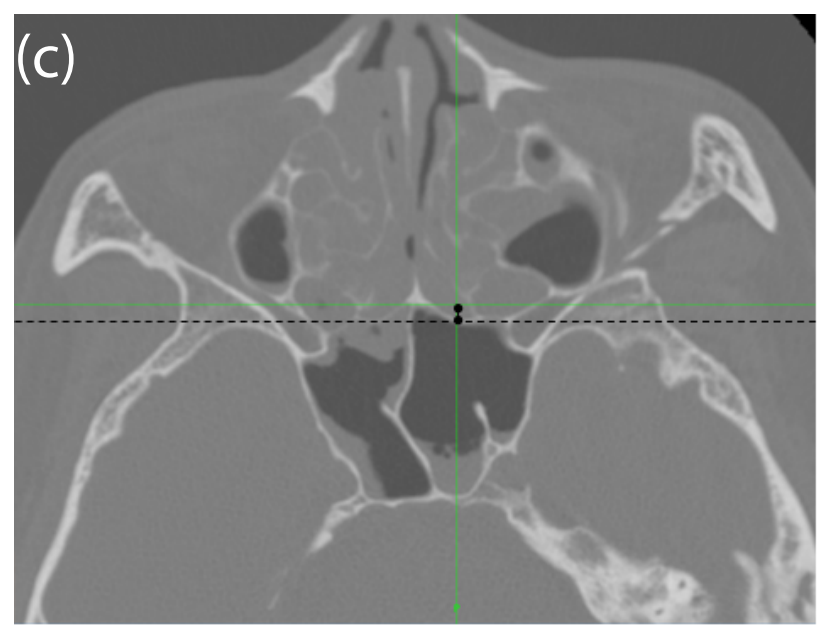

os

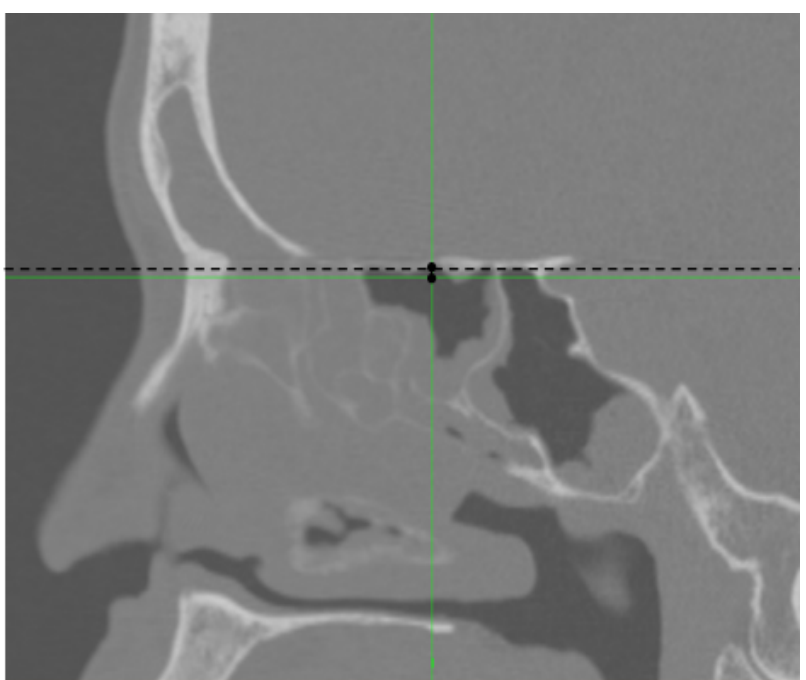

PE

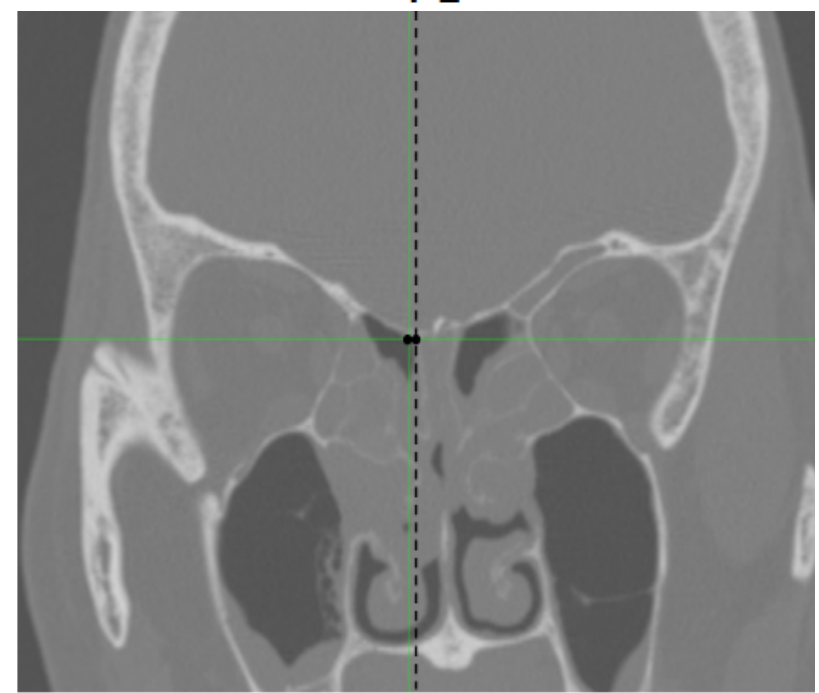

ST

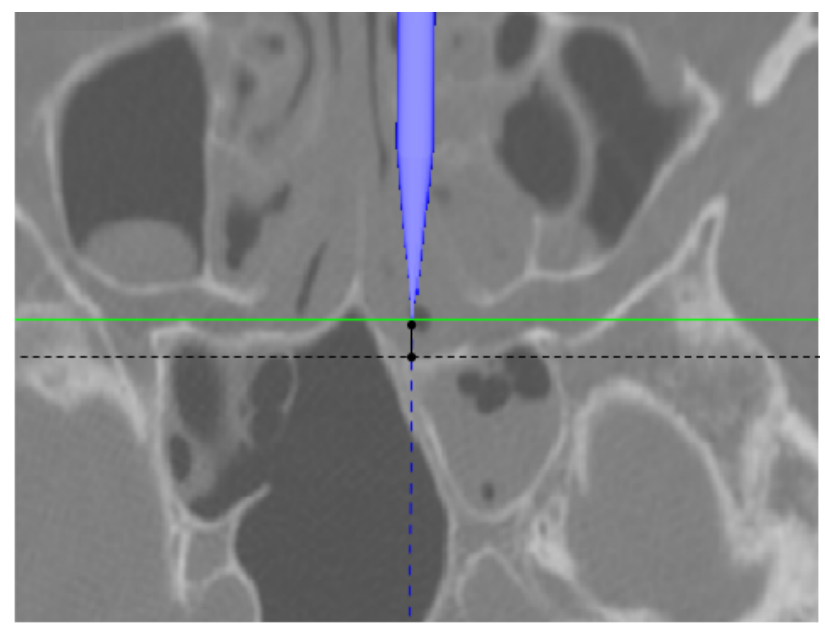

UC

Figure 1. The measurements of the navigation errors at (a) the central points of the anterior and posterior ethmoid roofs ( $A E$, PE, respectively), (b) the most medial point of the lamina papyracea (LP) and the insertion point of the superior turbinate (ST) and (c) the opening of the sphenoid sinus (OS) and the upper border of choana (UC). Dotted lines indicate imaginary lines passing the predetermined CT landmarks and perpendicular to the measuring axes. 
and sphenoid sinus. A navigational probe was then used to determine the NE. The probe was used to touch a predetermined anatomic landmark in the patient. The NE was measured as the vertical distance from the navigated tip of the probe (i.e. the crosshairs) to an imaginary plane passing the predetermined CT landmark and perpendicular to the measuring axis (Figure 1) $(4,10,13,16.17)$. The NE in the cranial-caudal (CC) direction was measured on the sagittal plane using the central points of the anterior and posterior ethmoid roofs ( $\mathrm{AE}, \mathrm{PE}$, respectively) as the predetermined CT landmarks, whereas the NE in the mediallateral (ML) direction was measured on the coronal plane with the most medial point of the lamina papyracea (LP) and the insertion point of the superior turbinate (ST) as the CT landmarks. The NE in the anterior-posterior (AP) direction was measured on the axial plane of the $\mathrm{CT}$ scan relative to the opening of the sphenoid sinus (OS) reference points and the upper border of choana (UC). The reason why predetermined landmarks were chosen is because these landmarks are easy to be identified on the specific planes of the $C T$ images. For example, the referred points of $A E$ and $P E$ are easier to be located on the sagittal plane than the coronal or the axial plane. However, even when we measure the navigation error being zero in one axis, it only means that the navigated point is equal to the predetermined virtual point in that axis, but the error may still exist in other axes. For all patients, the distance measurements were performed 3 times without repeating the registration process. The mean of these values was considered the NE for specific locations. All the NE results were expressed as absolute values for the purpose of statistical analysis.

\section{Statistical methods}

The times for the surface registration and surgical procedure on each side, as well as the NE in the AP, CC and ML directions, are expressed as mean values \pm standard deviations. The times used for the registration and surgery, Lund Mackey score for each group, as well as NEs in the specific anatomic locations of both groups were compared by independent t-test. In addition, oneway ANOVA followed by the Bonferroni test was employed for multiple comparisons of NEs among the 6 anatomic locations in the optic or electromagnetic navigation system. The level of significance was set at $p<0.05$. The statistical package SPSS 16.0 for Windows was used for all statistical analyses.

\section{Ethical considerations}

The institutional review board of Far Eastern Memorial Hospital approved this study, and written informed consent was obtained from all of the patients.

\section{Results}

Thirty patients aged between 20 and 62 years (mean 42 years) underwent bilateral endoscopic sinus surgery. The mean score on the Lund-Mackay CT scan classification system for all of the patients were 19 points. As regards to the severity of disease in both groups, there was no significant difference of unilateral Lund Mackey score between these 2 groups ( $9.7 \pm 1.5$ in the optic group v.s. $9.3 \pm 1.5$ in the electromagnetic group). The times required for surface registration and surgical procedure are $58.9 \pm 4.2$ seconds and $62 \pm 11.5$ minutes in the optic navigation group, and $33.2 \pm 2.4$ seconds and $56.7 \pm 11.1$ minutes in the electromagnetic group, respectively. The time for surgical procedure has no significant difference between these 2 groups; however, the time for surface registration was significantly longer in the optic navigation group than electromagnetic group $(p<0.05$, independent $t$-test). There were no cases of operative complications. In the optic navigation group, the NEs of $A E, P E$, LP, ST, OS and UC were $1.7 \pm 1.0,1.5 \pm 0.9,0.6 \pm 0.4,0.6 \pm 0.5,1.2$ \pm 0.7 and $1.4 \pm 0.8 \mathrm{~mm}$, respectively, with a significant difference among these 6 groups (Figure2) $(p<0.05$, one-way ANOVA test). In the electromagnetic navigation group, the NEs of $A E, P E, L P$, ST, OS and UC were $1.3 \pm 0.7,1.2 \pm 0.8,0.6 \pm 0.6,0.7 \pm 0.5,1.1 \pm$ 0.6 and $1.4 \pm 0.6 \mathrm{~mm}$, respectively, with a significant difference among these 6 groups (Figure 3 ) ( $p<0.05$, one-way ANOVA test). Among the 6 anatomic locations, the distance deviations in the ST and LP groups were significantly less than those in the other groups ( $p<0.05$, Bonferroni test) in both the optic (Figure 2) and electromagnetic (Figure 3) navigation setting. No significant differences were observed in any paired comparison between the NP, OS, AE and PE groups ( $p>0.05$, Bonferroni test). There was also no significant difference between the NE in the ST and LP groups ( $p>0.05$, Bonferroni test). Furthermore, in comparison to the NE in each specific location, there was no significant difference between the optical and electromagnetic navigation groups ( $p>0.05$, independent t-test).

\section{Discussion}

Over the past decade, image-guided surgical navigation has been welcomed as a technology in both primary and revision sinus surgery. The anatomic aid provided by navigation system is very important for the surgeon. It facilitates better intraoperative orientation and enables more thorough eradication of diseased tissue, especially in cases of extensive polyposis, revision surgery and neoplastic sinonasal disease.

Due to the delicate neighboring structures in close proximity to the orbits, vessels and base of the skull, a variety of major surgical complications, including loss of vision, diplopia, injury to the internal carotid artery, cerebrospinal fluid leak and brain damage may occur when the surgeon is unaware of which anatomical areas are dangerous ${ }^{(2,18)}$. The demand for safety can be particularly challenging in cases of anatomical complexity, mostly in patients with extensive sinus disease or requiring 


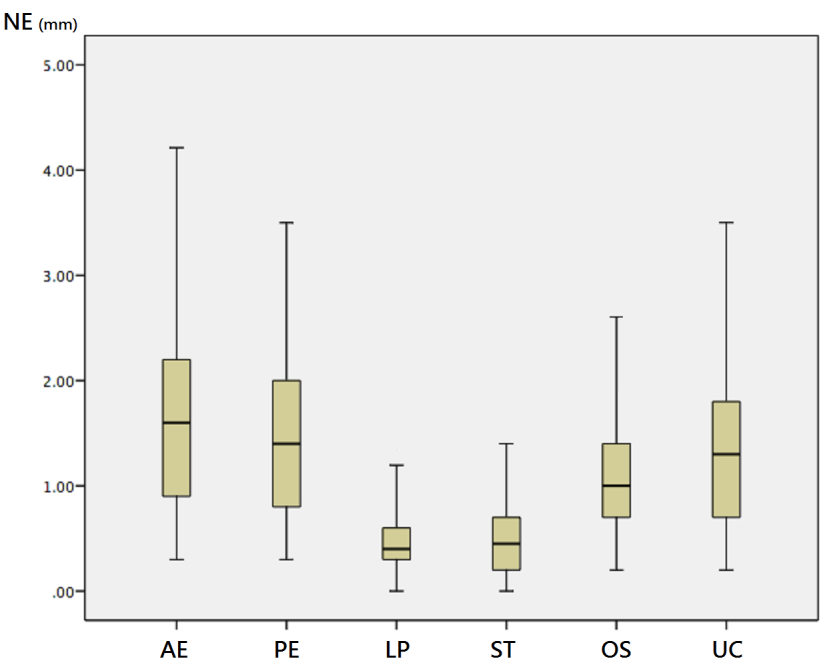

Figure 2. The navigation error (NE) of the optic navigation system in the 6 location groups. The NE in LP and ST groups (medial-lateral axis) was significantly less than that in the other 4 location groups (cranial-caudal and anterior-posterior axes). The bottom and top of the box are the first and third quartiles, and the band inside the box is the median. The ends of the whiskers represent the minimum and maximum of all of the data.

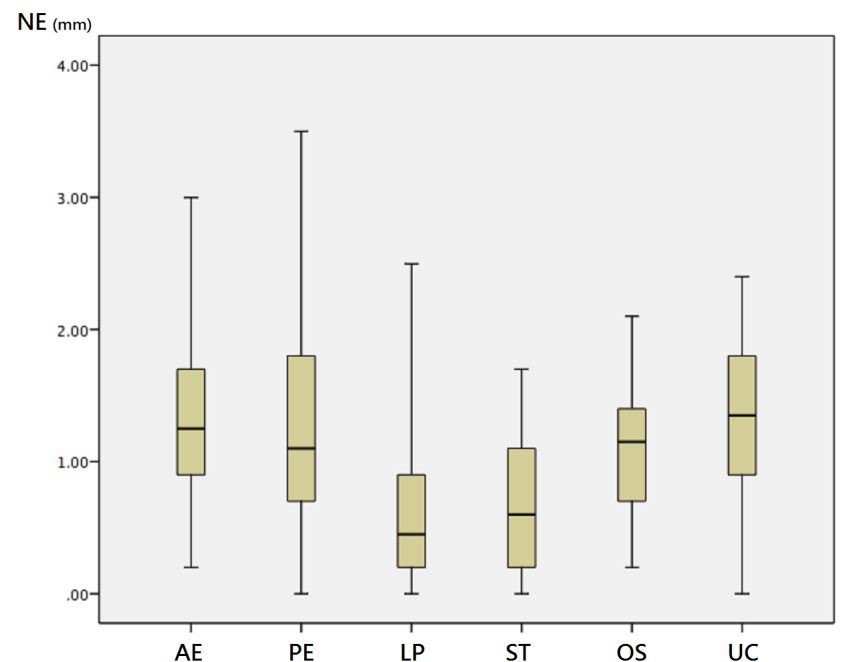

Figure 3. The navigation error (NE) of the electromagnetic navigation system in the 6 location groups. The NE in LP and ST groups (mediallateral axis) was significantly less than that in the other 4 location groups (cranial-caudal and anterior-posterior axes). The bottom and top of the box are the first and third quartiles, and the band inside the box is the median. The ends of the whiskers represent the minimum and maximum of all of the data. revision surgery. With the assist of this modern technology, surgeons may prevent accidental damage to vital structures in proximity to the surgical area by monitoring the real-time position of surgical instruments $(4,13,19,20)$.

The tracking modalities, registration methods and navigation locations all influence system accuracy. Hence, exact knowledge about the navigation systems under specific conditions is required for a confident use of CAS. Two main types of navigation modalities are in general use in current clinical practice. First, optical navigation systems use active light-emitting diodes or passive reflecting spheres that are mounted on the navigational instruments and the reference headset to detect the movement of the instruments by triangulation of an infrared camera system. Many studies have shown that optical navigation systems possess a high degree of accuracy for clinical use $(4,10,13,16)$. However, a major drawback of the optic navigation system is that no obstacle can be allowed between the infrared camera system and the navigational instruments ${ }^{(4)}$. Restated, continuous navigation may frequently be disturbed due to a blockage of the direct line of sight. Second, electromagnetic navigation systems do not need to keep the direct line of sight between all components because a different tracking technique is employed. While moving, the coil arrays embedded in the trackers can sense the change in the electromagnetic field that is constructed by an emitter. As a result, an electromagnetic tracking system can offer continuous localization information on the monitor without in- terruption throughout the operation and is superior to an optic tracking system in a cluttered operating field ${ }^{(10,14)}$.

In order to eliminate the need for the time-consuming application of the landmark and fiducial marker matching registration, a surface contour-based registration technique was proposed many years ago ${ }^{(11-13)}$. Although surface registration was shown to be less accurate as compared to the other registration methods ${ }^{(11,12,21)}$, the NEs using the former registration for both the optic and electromagnetic navigation systems are still within the range that are typically deemed sufficient for clinical purposes ${ }^{(21-23)}$. Because of small amount of registration time relative to the entire operation time $(1.58 \%$ in the optically navigated surgery and $0.98 \%$ in the electromagnetically navigated surgery), surface registration offers a great deal of practical convenience in navigation preparation. Although surface registration costs significantly less time in the electromagnetic navigation group than the optic one, the entire surgical times in both groups were similar.

To the best of our knowledge, this is the first report to measure NEs using both optic and electromagnetic navigation systems on the same patient in live surgery. The results show that the NEs in the 6 measured areas have maximal mean values of 1.7 $\mathrm{mm}$ in the optic navigation group and $1.4 \mathrm{~mm}$ in the electromagnetic navigation group. This finding can be taken as acceptable accuracy for clinical practice, which is generally agreed to be less than $3 \mathrm{~mm}{ }^{(21-23)}$. At the time when the navigation 
system was first developed to assist endoscopic sinus surgery, the optical tracking system was widely adopted due to better accuracy than that of the electromagnetic system ${ }^{(10,24)}$. Using the same navigation system as ours, Kral et al. ${ }^{(10)}$ reported both the optic and electromagnetic navigation systems have excellent sub-millimetric accuracy in the case of anatomical specimens from an experimental setup. However, the optical tracking was reported to be significantly more precise than the electromagnetic tracking. In contrast, the NEs measured in our study demonstrated that the accuracy of electromagnetic tracking is comparable to that of optic tracking for live navigated endoscopic procedures. This may be because recent hardware and software advances in the electromagnetic navigation system have improved the system accuracy, which was initially highly influenced by ferromagnetic distortion ${ }^{(25,26)}$. Furthermore, the advantage of the admirable accuracy of the optic tracking system when used under optimized laboratory conditions is somewhat offset in the course of live surgery, leading to a certain loss of precision. The surface registration method in this study also played a role in a decrease in accuracy, because either a displacement of the reference frame or distortion of the facial contour caused by intraoperative stretching of the skin/soft tissue may result in a discordance between the facial contour and the preoperative image ${ }^{(11-13)}$. It may be appropriate to repeat surface registration to overcome the increased NEs in consideration of intraoperative anatomical drift. However, in this study, we performed surface registration at the beginning of surgery to evaluate whether the NEs remained acceptable throughout the surgery. All of these factors affect the navigational accuracy; consequently, our comparative study of these two systems used in the same patients provides objective evidence that both systems are similar in terms of accuracy, no matter which location of the NEs was measured. Together with previous reports in the existing literature ${ }^{(10-14,20,24,27)}$, our results and experience provide a referential basis that should prove to be informative for a surgeon or institution in the selection of a navigation system.

Furthermore, in terms of both optic navigation and electromagnetic navigation, our results show that the NEs in the ML direction had a significantly higher precision than NEs in the CC and AP directions. The reason for the difference lies mostly in the fact that a more precise correlation between the pre-operative image and intra-operative anatomy depends on the collection of more widely dispersed registration points, regardless of the modality of the navigation system. Because the extent of the divergence in tracing the points of the facial contour is greater for the ML axis than the CC and AP axes, it is more precise when analyzing the navigational accuracy in the ML direction. In addition, a shift of the patient's skin/soft tissue, mostly in the AP direction, or changes in the tension in the muscles of expression during the course of surface registration may also play a role in an invalid data set correlation for CAS, especially in the AP direction. Since the measurement has even less precision on the CC and AP axes, the surgeon has to keep in mind that dissection near the skull base presents greater risks than when performed closer to the lamina papyracea. A thorough knowledge of the complex anatomy and surgical techniques, instead of just relying on the navigation system, remains essential for performing safe endoscopic sinus surgery ${ }^{(28,29)}$, even though this study has confirmed that the NEs in both systems are quite acceptable in terms of providing assistance during the surgery.

\section{Conclusions}

The electromagnetic navigation system takes significantly less time to complete the surface registration than the optic navigation system. The time for registration in both systems only accounts for less than $2 \%$ of the time taken for the surgery on one side. Furthermore, the accuracy of the two navigation systems is acceptable and comparable in clinical use. However, we recommend using the electromagnetic navigation in CAS because of the advantage provided by continuous tracking without interruption, especially in cluttered operation theatres. In the three-dimensional analysis of the two-system accuracy, the best accuracy was measured in the ML direction compared with the other two axes. This finding suggests that surgeons should be more cautious when dissecting in the AP or CC direction during the course of navigation for sinus surgery.

\section{Author contributions}

CMC: wrote paper, analyzed and interpreted data,PWC: performed surgeries and supervised study.

\section{Conflict of interest}

None declared.

\section{References}

1. Kennedy DW, Zinreich SJ, Rosenbaum AE, Johns ME. Functional endoscopic sinus surgery. Theory and diagnostic evaluation. Arch Otolaryngol. 1985; 111: 576-582.

2. Mafee MF, Chow JM, Meyers R. Functional endoscopic sinus surgery: anatomy, CT screening, indications, and complications AJR Am J Roentgenol. 1993; 160: 735-744.
3. Schlöndorff G, Mösges R, Meyer-Ebrecht D, Krybus W, Adams L. CAS (computer assisted surgery). A new procedure in head and neck surgery. HNO. 1989; 37: 187-190.

4. Metson RB, Cosenza MJ, Cunningham MJ, Randolph GW. Physician experience with an optical image guidance system for sinus surgery. Laryngoscope. 2000; 110: 972-976.

5. Fried MP, Kleefield J, Gopal H, Reardon
E, Ho BT, Kuhn FA. Image-guided endoscopic surgery: results of accuracy and performance in a multicenter clinical study using an electromagnetic tracking system. Laryngoscope. 1997; 107: 594-601.

6. Mösges R1, Schlöndorff G. A new imaging method for intraoperative therapy control in skull-base surgery. Neurosurg Rev. 1988; 11: 245-247 
7. Reinhardt HF, Horstmann GA, Gratzl O Microsurgical removal of deep vascular malformations using sonar-stereometry. Ultraschall Med. 1991; 12: 80-83.

8. Maurer CR Jr, Maciunas RJ, Fitzpatrick JM Registration of head CT images to physical space using a weighted combination of points and surfaces. IEEE Trans Med Imaging. 1998; 17: 753-761.

9. Maurer CR Jr, Fitzpatrick JM, Wang MY, Galloway RL Jr, Maciunas RJ, Allen GS. Registration of head volume images using implantable fiducial markers. IEEE Trans Med Imaging. 1997; 16: 447-462.

10. Kral F, Puschban EJ, Riechelmann H, Pedross F, Freysinger W. Optical and electromag netic tracking for navigated surgery of the sinuses and frontal skull base. Rhinology 2011; 49: 364-368.

11. Raabe A, Krishnan R, Wolff R, Hermann E, Zimmermann M, Seifert V. Laser surface scanning for patient registration in intracranial image-guided surgery. Neurosurgery. 2002; 50: 797-803

12. Schlaier J, Warnat J, Brawanski A Registration accuracy and practicability of laser-directed surface matching. Comput Aided Surg 2002; 7: 284-290.

13. Ledderose GJ, Stelter K, Leunig A, Hagedorn $H$. Surface laser registration in ENT-surgery: accuracy in the paranasal sinuses--a cadaveric study. Rhinology. 2007; 45: 281-285.

14. Metson R, Gliklich RE, Cosenza M. A comparison of image guidance systems for sinus surgery. Laryngoscope. 1998; 108: 1164-1170.

15. Meltzer EO, Hamilos DL, Hadley JA, et al Rhinosinusitis: Developing guidance for clinical trials. Otolaryngol Head Neck Surg. 2006; 135: S31-80.

16. Claes J, Koekelkoren E, Wuyts FL, Claes GM, Van den Hauwe L, Van de Heyning
$\mathrm{PH}$. Accuracy of computer navigation in ear, nose, throat surgery: the influence of matching strategy. Arch Otolaryngol Head Neck Surg. 2000; 126: 1462-1466.

17. Chang CM, Fang KM, Huang TW, Wang CT, Cheng PW. Three-dimensional analysis of the surface registration accuracy of electromagnetic navigation systems in live endoscopic sinus surgery. Rhinology. 2013; 51: 343-348.

18. May M, Levine HL, Mester SJ, Schaitkin B. Complications of endoscopic sinus surgery: analysis of 2108 patients--incidence and prevention. Laryngoscope. 1994; 104: 10801083.

19. Tschopp KP, Thomaser EG. Outcome of functional endonasal sinus surgery with and without CT-navigation. Rhinology. 2008; 46: 116-120.

20. Hepworth EJ, Bucknor M, Patel A, Vaughan WC. Nationwide survey on the use of image-guided functional endoscopic sinus surgery. Otolaryngol Head Neck Surg. 2006; 135: 68-73.

21. Knott PD, Batra PS, Butler RS, Citardi MJ. Contour and paired-point registration in a model for image-guided surgery. Laryngoscope. 2006; 116: 1877-1881.

22. Metson R. Image-guided sinus surgery: lessons learned from the first 1000 cases. Otolaryngol Head Neck Surg. 2003; 128: 8-13.

23. Roth M, Lanza DC, Zinreich J, Yousem D, Scanlan KA, Kennedy DW. Advantages and disadvantages of three-dimensional computed tomography intraoperative localization for functional endoscopic sinus surgery. Laryngoscope. 1995; 105: 1279-1286.

24. Ecke U, Luebben B, Maurer J, Boor S, Mann WJ. Comparison of Different ComputerAided Surgery Systems in Skull Base Surgery. Skull Base. 2003; 13: 43-50.
25. Hummel J, Figl M, Birkfellner W, et al. Evaluation of a new electromagnetic tracking system using a standardized assessment protocol. Phys Med Biol. 2006; 51 : N205-210.

26. Yaniv Z, Wilson E, Lindisch D, Cleary K. Electromagnetic tracking in the clinical environment. Med Phys. 2009; 36: 876-892.

27. Stelter K, Andratschke M, Leunig A Hagedorn $\mathrm{H}$. Computer-assisted surgery of the paranasal sinuses: technical and clinical experience with 368 patients, using the Vector Vision Compact system. J Laryngol Otol. 2006; 120: 1026-1632.

28. Stelter K, Ertl-Wagner B, Luz M, et al. Evaluation of an image-guided navigation system in the training of functional endoscopic sinus surgeons. A prospective, randomised clinical study. Rhinology. 2011; 49: 429-437.

29. Eliashar R, Sichel JY, Gross M, et al. Image guided navigation system-a new technology for complex endoscopic endonasal surgery. Postgrad Med J. 2003; 79: 686-690.

\section{Po-Wen Cheng, MD}

Department of Otolaryngology

Far Eastern Memorial Hospital

21, Sec.2, Nanya S. Rd

Banqiao Dist.

New Taipei City

Taiwan

Tel: + 886-2-89667000 ext 2833

Fax: + 886-2-77282149

E-mail: powenjapan@yahoo.com.tw 